\title{
A New Analytical Model for the Analysis of Economic Processes
}

\author{
Paolo Di Sia \\ Faculty of Economics and Management and Faculty of Education, Free University of Bozen-Bolzano, \\ Bolzano, Italy \\ Email: paolo.disia@yahoo.it
}

Received May 29, 2013; revised June 29, 2013; accepted July 16, 2013

Copyright (C) 2013 Paolo Di Sia. This is an open access article distributed under the Creative Commons Attribution License, which permits unrestricted use, distribution, and reproduction in any medium, provided the original work is properly cited.

\begin{abstract}
In this work it is presented a new powerful model, well checked in the last years with the analysis of the dynamics of nano-bio-structures. The model permits the detailed study of the dynamical evolution of systems also at macro-level, offering analytical relations concerning the velocity and the distance of an economic variable, so as its diffusion in time; it permits the analysis of economic processes in general. In this paper it will be focused in particular about economic cycles.
\end{abstract}

Keywords: Economic Processes; Mathematical Modelling; Diffusion; Dynamics; Econophysics

\section{Introduction}

Theories of economic cycles regard capitalist economic systems and are characterized by a not uniform development, but rather intrinsically marked by oscillations. Fluctuations in pre-capitalist systems were due to external factors to the economic sphere, such as the harvest variability, the consequences of wars or epidemics, and so on. The expansion of the capitalist market and the socio-economic and institutional resulted changes had the effect of a significant change of the oscillation characteristics. The cyclic evolution was determined in increasingly way by endogenous factors and peculiar propagation mechanisms to the capitalist system; the nature of the cyclic processes changed so in essential manner [1].

This new type of oscillation could be due by the development of the banking system and by its ability to create credit. The treatment of models of cyclic development requires the use of complex mathematical techniques for analyzing the modifications of structural nature, which characterize the processes of development; often they occur in slow but continuous changes and produce variations, that, cumulatively, become increasingly relevant in relation to the crisis. The cyclic trends are able to exert a strong influence on the short-term dynamics, but they can with the same speed change direction. The integrated analysis of these differing movements is complicated by the need to take into account not only of the trends of strictly economic variables, but also of social, political and institutional variables. The established inter-relationships between all these variables could not be neglected when we want to go beyond the horizon of short-term time periods [2]. The articulated, but productive way of interaction between economics and physics reflects the extensive reality of the mutual influence of various disciplines through the complexity theory and other trans-disciplinary theories, such as the non-linear dynamics, the game theory and other mathematically sophisticated approaches [3].

\section{Indicators and Cycle Characteristics}

One of the most widely used economic indicators is given by the performance of gross domestic product (GDP) in real terms, which succinctly measures the fluctuations in production at different dates. It depends on many variables, as the final consumptions, the statal spending, investments, imports, exports, etc $[4,5]$.

Other indicators of partial phenomena are the trend of production in the different sectors, levels of employment, investments, monetary aggregates and credit, applications for specific products, consumption of raw materials, prices, profits (Figure 1).

The performances of the different indicators are not identical, but in many cases quite similar. There is a remarkable agreement of different indicators in signaling periods of acceleration and slowing of production. 


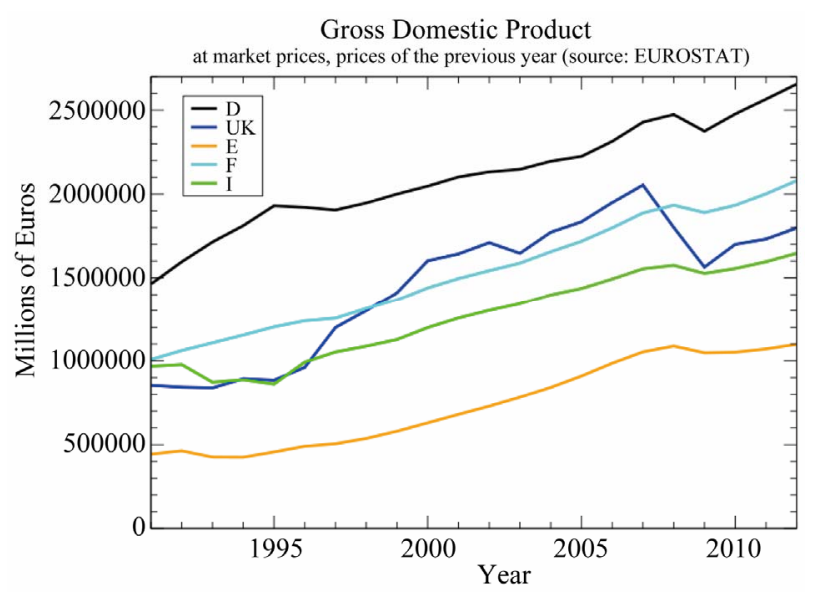

Figure 1. Gross domestic product of five major economies of the European community since 1991 (In million Euro (or ECU), official data from EUROSTAT) [6].

The study of these changes can be crucial to explain the relationship between cycles and development [7].

The trend of the time series is usually rough, it is not easy to recognize cyclic regularity. It needs a work of cleaning the raw data, firstly to eliminate those components that have a cyclic explanation as purely seasonal (for example the decline of industrial production in August) or accidental (as monthly variations of production caused by the different number of days working), and then also to eliminate patterns requiring a special "ad hoc" explanation, determined by important but unique events, as the effects of war, natural disasters $[8,9]$. The relationship between cycle and growth is a phenomenon coexisting in the representation that the empirical data provide. The majority of economists decided to solve the problem by making a clear separation of the two phenomena. The statistical methods used for this purpose differ in details, but in essence all consist in the evaluation of a trend, which should give an account of growth, and in the calculation of variances, that the considered time series show against the trend. An example, with a linear trend, is shown in Figure 2.

A substantially always increasing trend can be decomposed into two components: one showing a uniform growth for a long period of time and the other that instead oscillates, assuming positive or negative values in relation on whether of the considered variable overtakes the long term trend. Normally it is assumed that the trend represents the performance of system equilibrium and that cyclical deviations are due to deviations. The trend calculation is of necessity made on the basis of data of the past. But the present and the future may have very different characteristics from those of the past, especially if there are modifications of structural character, which is more probable as longer is the period of data observation [10].

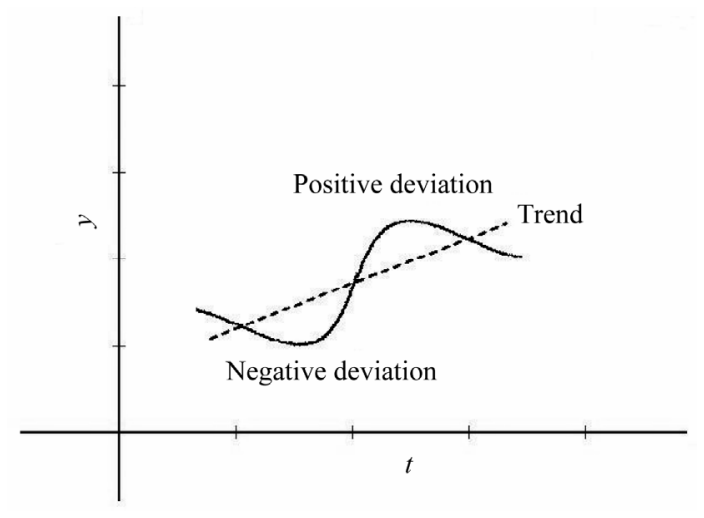

Figure 2. Possible variations of a linear trend.

Looking at the profile of the deviations, the identification of the phases, that usually describe the cycle, becomes easy: depression, resumption, boom, recession (Figure 3).

The amplitude of booms and depression of historical cycles had not always comparable size, so as their duration was also highly variable. Therefore, some modern theorists begun to doubt the very existence of phenomena, that can be properly called cyclic [11]. Every historical cycle has its own characteristics because it takes place in different and changing socio-economic situations and is influenced by a different measure extent with endogenous (economic) and external (non-economic) factors [12-14]. The various indicators begin to move with greater velocity, the recovery spreads and becomes boom. It grows the production, sales, employment, real income, used production capacities, applications for raw materials and finished products, the quantity of money, its velocity of circulation, the amount of credit and loans to economy. However the boom does not last indefinitely, economic activity first begins to decelerate and then to contract.

\section{Theoretical Explanations of Cycles}

The reflection on the causes and mechanisms that generate cyclic movements has a long history. Many of the theories of the cycle, that dominate the scene, have still now their antecedents in formulations which have appeared in the last century or the beginning of it. The following treatments have benefited of a more rigorous analytical framework and a much larger amount of empirical knowledge.

The first explanation of commercial crises is traced to the effects of exogenous factors: the variability of crops wars, exploitation of new gold deposits at the opening or closing of foreign markets, but also technical innovation (Lord Overstone in 1837 and Thomas Tooke in 1838) $[15,16]$. In the last century, the crises assumed often financial characteristics. Already Overstone and Tooke emphasized how the fluctuations of credit counted for 


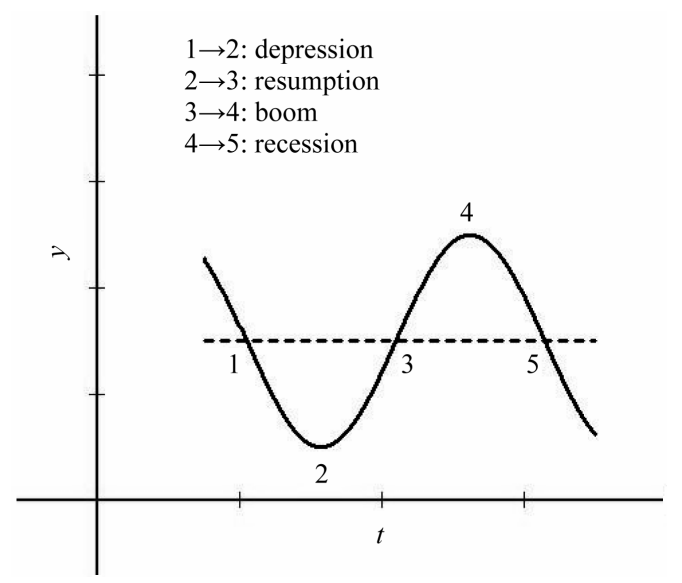

Figure 3. The various levels of a displacement by a trend.

much in exalting the phases of expansion and in aggravating that of contraction.

Later, John Stuart Mill pointed out the influence of speculation and the forecast errors of the operators that caused irrational fluctuations in the demand for credit, which, consequently, had the effect of enhancing the cyclic phenomena. At the beginning of the last century, the different theories explained the crisis in terms of under-consumption and savings excess [17].

It has seen that, in the case of damped oscillations, the permanence of the cycle time can be ensured by a succession of irregular, random nature shocks. Theories based on the interaction between multiplier and accelerator did not give exclusive importance on this fact [18]. On the other hand, the empirical investigations seem to suggest that the values of the parameters of the model are such as to give rise to a solution of "explosive" type, i.e. to oscillations or amplified in exponential growth. The most well-known theories have recognized this peculiarity, but have introduced upper and lower limits, which block the divergent trends of the system bouncing in the opposite directions [19].

Explosives trends occur at high values of the accelerator. With this increase in demand (exogenous), it follows a significant increase in investment and thus a rapid growth. The boom is self-fueled and the system is pushed towards the upper limit by full capacity utilization (especially in sectors producing capital goods) or by the full employment of labor (with the required characteristics). The introduction of lower and upper limits allowed for a greater realism of the theory and has eliminated some important deficiencies. Richard M. Goodwin introduced the ideas of ceilings and floors [20], John R. Hicks an exogenous trend of growth for individual investments [21]. The introduction of lower and upper limits made non-linear the original model; the accelerator does not work as before when limits are reached. This is the advantage of the model, but also a limit, because at linear level cycle disappears or becomes explosive.

\section{A New Derived Model}

A recent theoretical analytical formulation showed to fit very well experimental scientific data and offers interesting new predictions of various peculiarities in nanostructures [22-24].

The new model was utilized at today at scale length of order of nanometers. But the de Broglie thermal wavelength refers to the dual nature of reality. Associating the wavelength to the impulse through the de Broglie relation, it is possible to define a thermal wavelength $\lambda_{t}$ for every object at macrolevel via the relation:

$$
\lambda_{t}=h / \sqrt{3 m k_{B} T}
$$

In Equation (1), $h$ is the Planck constant, $k_{B}$ the Boltzmann's constant, $m$ and $T$ mass and temperature of the considered system respectively. With this gauge factor it is so possible to study the dynamics of reality processes presenting oscillations in time, so as characteristics of diffusivity in time.

The model offers the analytical formulation of the most important quantities related to the dynamics of a generic process, i.e.:

a) The velocities correlation function:

$$
\langle\boldsymbol{v}(t) \cdot \boldsymbol{v}(0)\rangle_{T}
$$

at temperature $T$, from which the velocity $v(t)$ is obtainable;

b) The mean squared deviation of position:

$$
R^{2}(t)=\left\langle[\boldsymbol{R}(t)-\boldsymbol{R}(0)]^{2}\right\rangle
$$

from which the position vector $R(t)$ at the time $t$ is obtainable;

c) The time-dependent diffusion $D(t)$ of the system:

$$
D(t)=1 / 2\left(\mathrm{~d} R^{2}(t) / \mathrm{d} t\right)
$$

Equations (2)-(4) are fundamental in deducing the most important characteristics concerning the transport phenomena of processes.

The roots of the model lie in the linear response theory and follow the standard time-dependent approach; the conductivity $\sigma(\omega)$ is in general a complex function of the frequency $\omega$ and can be deduced from linear response theory. The model is based on a complete Fourier transform of the frequency-dependent complex conductivity $\sigma(\omega)$ of the system; this is in general a complex function of the frequency $\omega$, which can be deduced from linear response theory (Green-Kubo formula):

$$
\sigma_{\beta \alpha}(\omega)=\left(e^{2} / \hbar V\right) \int_{0}^{\infty} \mathrm{d} t \mathrm{e}^{\mathrm{i} \omega t} \int_{0}^{\beta} \mathrm{d} \lambda\left\langle\boldsymbol{v}^{\alpha}(t-i \lambda) \boldsymbol{v}^{\beta}(0)\right\rangle
$$

By inversion of Equation (5), it is possible to find the 
velocities correlation function $\langle\boldsymbol{v}(t) \cdot \boldsymbol{v}(0)\rangle_{T}$ inside the integral. The presence of an integration from 0 to $\infty$ is however a problem for the analytical inversion, but it can be overcame evaluating the integral on the entire time axis $(-\infty,+\infty)$. Considering the real part of the complex conductivity in Equation (5), the extension to the entire time axis is possible and a complete Fourier transform can be performed, obtaining directly real velocities. The integral can be resolved in the complex plane considering a Cauchy integration; as a result, the velocities correlation function can be evaluated exactly by the residue theorem [25]. After this step, it is possible to obtain the analytical form of the mean square deviation of position (Equation (3)) and of the diffusion coefficient $D(t)$ (Equation (4))

The deduced results are, with $x=t / \tau$ :

$$
\begin{aligned}
&\langle\boldsymbol{v}(0) \cdot \boldsymbol{v}(t)\rangle=(K T / m) \exp (-x / 2) \\
& \cdot\left(\cos \left(\left(\alpha_{R} / 2\right) x\right)-\left(1 / \alpha_{R}\right) \sin \left(\left(\alpha_{R} / 2\right) x\right)\right) \\
& R^{2}(t)=(\left.2 K T / m \omega_{0}^{2}\right)\left(-\left(1 / \alpha_{R}\right) \sin \left(\left(\alpha_{R} / 2\right) x\right) \exp (-x / 2)\right. \\
&-\left.\cos \left(\left(\alpha_{R} / 2\right) x\right) \exp (-x / 2)+1\right) \\
& D(t)=(K T / 2 m)\left(\left(4 \tau^{2} / \alpha_{R}\right) \sin \left(\left(\alpha_{R} / 2\right) x\right) \exp (-x / 2)\right)
\end{aligned}
$$

Equations (6)-(8) are related to a first model constant $\alpha_{R}=\sqrt{4 \tau^{2} \omega_{0}^{2}-1} ; \alpha_{R}$ is a real non-negative number. In the same way the model contains three analytical relations related to the second model constant

$\alpha_{I}=\sqrt{1-4 \tau^{2} \omega_{0}^{2}} ; \alpha_{I} \in[0,1]$ and real. One of the most interesting peculiarities of the model is the "time domain" used approach, not previously found in such a contest, contrarily to the existing theoretical approaches of literature, which are "frequency domain" treatments and/or numerical methods [22-24].

The model was tested with the most utilized nanomaterials at today and describes transport properties of nano- and bio-materials [26-33], including previous important models [34] and offering new interesting peculiarities, still not experimentally found.

The analytical form of previous cited relations is composed by a superposition of exponentials and/or products among exponentials and sinusoidal (cosinusoidal) functions, giving rise to trends as indicated in Figures 4-6.

The model is so related to variables as the temperature, the energy and the mass, the weight of the various states (at quantum level), which, "mutatis mutandis", are very important in a lot of economic processes too, from economic geography and gravity models for economy, to economic behaviours treated with game theory [35], eco-

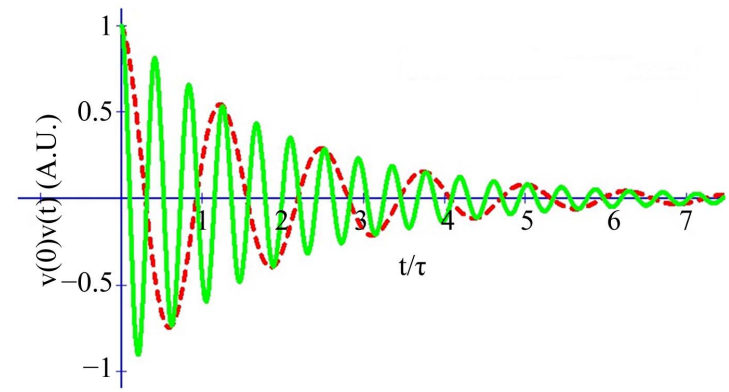

Figure 4. Velocities correlation function vs $t / \tau$ for two values of the parameter of the model $\alpha_{R}=\sqrt{4 \tau^{2} \omega_{0}^{2}-1}$. We note clear damped oscillations in time [22-24]. $\alpha_{R}=10 \rightarrow$ red line; $\alpha_{R}=\mathbf{3 0} \rightarrow$ green line.

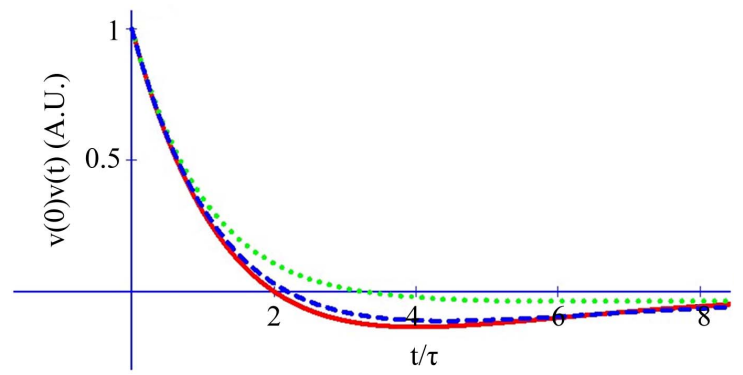

Figure 5. Positive and negative deviation by a linear trend (represented in this case by $t$-axis) for some values of the parameter of the model $\alpha_{I}=\sqrt{1-4 \tau^{2} \omega_{0}^{2}} \quad[22-24] . \alpha_{I}=0.1$ $\rightarrow$ red line; $\alpha_{I}=0.5 \rightarrow$ blue line; $\alpha_{I}=0.9 \rightarrow$ green line.

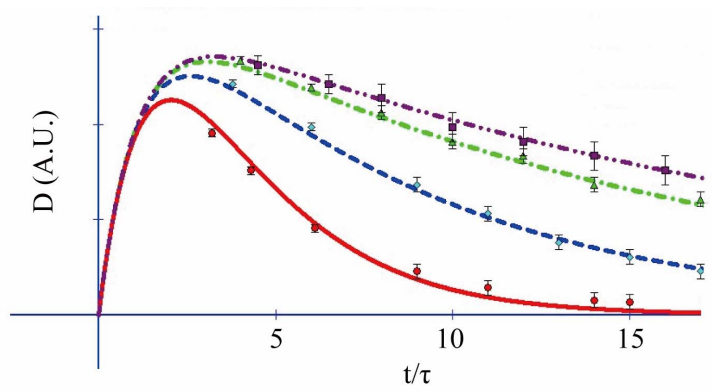

Figure 6. Behaviour of the diffusion $D$ for $\alpha_{I}$ varying in its definition interval [22-24], with $\tau=10^{-12} \mathrm{~s}$. Dots with error bars represent experimental data. $\alpha_{I}=0.25 \rightarrow$ red line; $\alpha_{I}=0.75 \rightarrow$ blue line; $\alpha_{I}=0.85 \rightarrow$ green line; $\alpha_{I}=0.95 \rightarrow$ violet line.

nomic analogies to thermodynamics and chaos in business cycle theories $[36,37]$.

The relations between physical and economic variables have been in the past always of great interest; as above introduced, we can think to gravity models of trade in international economics, so as in social science, where the physical variables are related to correspondent economic variables, as $F$ to the trade flow, $M$ to the economic mass of each country, $R$ to the distance. Gravity 
models have also been used in international relations for the evaluation of the impact of treaties and alliances on trade, so as for testing the effectiveness of trade agreements and organizations.

For all these reasons, the indicated model can effectively be an interesting technical tool for the implementation of economic data and the investigation of new economic peculiarities.

\section{Conclusion}

The strength of the new derived model consists of its ability to fit very well experimental data, accommodating not completely understood behaviours and including previous models, like the Smith model. The possibility to work at macrolevel through the use of the gauge factor (1) allows interesting applications in any sector in which velocities, distancies, oscillations and diffusion are involved. The started tests with economic data are giving favorable evidence of an interesting assistance of this model in the interpretation and comprehension of many phenomena at economic level.

\section{REFERENCES}

[1] G. Dosi and R. R. Nelson, "An Introduction to Evolutionary Theories in Economics," Journal of Evolutionary Economics, Vol. 4, No. 3, 1994, pp. 153-172. doi:10.1007/BF01236366

[2] J. T. Cuddington and C. M. Urzúa, "Trends and Cycles in the Net Barter Terms of Trade: A New Approach," Economic Journal, Vol. 99, No. 396, 1989, pp. 426-442. doi: $10.2307 / 2234034$

[3] P. Di Sia, "Nature and Development of Econophysics: A Short Report," Theoretical Economics Letters, Vol. 2, No. 2, 2012, pp. 183-185. doi:10.4236/tel.2012.22032

[4] "Measuring the Economy, a Primer on GDP and the National Income and Product Accounts,” 2007. www.bea.gov

[5] http://www.hm-treasury.gov.uk/data_gdp_backgd.htm

[6] http://it.wikipedia.org/wiki/File:GDP_EU_TOP_5.svg

[7] M. Hallet, "Economic Cycles and Development Aid: What Is the Evidence from the Past?” European Commission, Directorate-General for Economic and Financial Affairs, Issue 5, 2009.

[8] C. Cohen and E. D. Werker, "The Political Economy of 'Natural' Disasters," Journal of Conflict Resolution, Vol. 52, No. 6, 2008, pp. 795-819. doi:10.1177/0022002708322157

[9] D. Bergholt and P. Lujala, "Climate-Related Natural Disasters, Economic Growth, and Armed Civil Conflict," Journal of Peace Research, Vol. 49, No. 1, 2012, pp. 147-162. doi:10.1177/0022343311426167

[10] V. Zarnowitz and A. Ozyildirim, "Time Series Decomposition and Measurement of Business Cycles, Trends and Growth Cycles,” Journal of Monetary Economics, Vol.
53, No. 7, 2006, pp. 1717-1739. doi:10.1016/j.jmoneco.2005.03.015

[11] T. Puu, "Attractors, Bifurcations \& Chaos: Nonlinear Phenomena in Economics,” Springer-Verlag, Berlin, Heidelberg, 2003.

[12] P. Kennedy, “A Guide to Econometrics," 6th Edition, Wiley-Blackwell, Hoboken 2008.

[13] M. Verbeek, “A Guide to Modern Econometrics," 4th Edition, Wiley, New York, 2012.

[14] T. Todorova, "Problems Book to Accompany Mathematics for Economists,” John Wiley \& Sons, Inc., New York, 2012.

[15] A. M. Andreades, "History of the Bank of England," 4th Edition, Frank Cass \& Co. Ltd., London, 1966.

[16] J. Francis, "History of the Bank of England, Its Times and Traditions, from 1694 to 1844,” Banker’s Magazine, New York, 1862.

[17] D. F. Thompson, “John Stuart Mill and Representative Government,” Princeton University Press, Princeton, 1976.

[18] C. L. Evans and D. A. Marshall, "Fundamental Economic Shocks and the Macroeconomy," Journal of Money, Credit and Banking, Vol. 41, No. 8, 2009, pp. 1515-1555. doi:10.1111/j.1538-4616.2009.00271.x

[19] W. Semmler, "On Nonlinear Theories of Economic Cycles and the Persistence of Business Cycles,” Mathematical Social Sciences, Vol. 12, No. 1, 1986, pp. 47-76. doi:10.1016/0165-4896(86)90047-8

[20] V. Velupillai, “Richard Goodwin: 1913-1996,” Economic Journal, Vol. 108, No. 450, 1998, pp. 1436-1449. doi:10.1111/1468-0297.00351

[21] J. R. Hicks, “Classics and Moderns: Vol. III of Collected Essays in Economic Theory,” Basil Blackwell, Oxford, 1983.

[22] P. Di Sia, “Classical and Quantum Transport Processes in Nano-Bio-Structures: A New Theoretical Model and Applications,” PhD Thesis, Verona University, 2011.

[23] P. Di Sia, “An Analytical transport Model for Nanomaterials," Journal of Computational and Theoretical Nanoscience, Vol. 8, No. 1, 2011, pp. 84-89. doi:10.1166/jctn.2011.1663

[24] P. Di Sia, “An Analytical Transport Model for Nanomaterials: The Quantum Version,” Journal of Computational and Theoretical Nanoscience, Vol. 9, No. 1, 2012, pp. 3134. doi:10.1166/jctn.2012.1992

[25] W. Rudin, "Real and Complex Analysis,” McGraw-Hill International Editions: Mathematics Series, McGraw-Hill Publishing Co., 1987.

[26] P. Di Sia, "New Theoretical Results for High Diffusive Nanosensors Based on ZnO Oxides,” Sensors and Transducers Journal, Vol. 122, No. 1, 2010, p. 1.

[27] P. Di Sia, “Oscillating Velocity and Enhanced Diffusivity of Nanosystems from a New Quantum Transport Model,” Journal of Nano Research, Vol. 16, 2011, pp. 49-54. doi:10.4028/www.scientific.net/JNanoR.16.49

[28] P. Di Sia, “A New Theoretical Method for Transport Processes in Nanosensoristics,” Journal of Nano Research, Vol. 20, 2012, pp. 143-149. 
doi:10.4028/www.scientific.net/JNanoR.20.143

[29] P. Di Sia, "Nanotechnology between Classical and Quantum Scale: Application of Anew Interesting Analytical Model,” Applied Surface Science, Vol. 5, No. 1, 2012, p. 1.

[30] P. Di Sia, “About the Influence of Temperature in SingleWalled Carbon Nanotubes: Details from a New DrudeLorentz-Like Model,” Applied Surface Science, Vol. 275, 2013, pp. 384-388. doi:10.1016/j.apsusc.2012.10.132

[31] P. Di Sia, “A New Theoretical Model for the Dynamical Analysis of Nano-Bio-Structures," Advances in Nano Research, Vol. 1, No. 1, 2013, p. 1.

[32] P. Di Sia, "Characteristics in Diffusion for High-Efficiency Photovoltaics Nanomaterials: An Interesting Analysis,” Journal of Green Science and Technology, 2013, in press.

[33] P. Di Sia, “A New Drude-Lorentz-Like Model for Rela- tivistic Particles,” Accepted for “3rd International Conference on Theoretical Physics, Theoretical Physics and Its Application, Moscow, 24-28 June 2013.

[34] N. V. Smith, "Classical Generalization of the Drude Formula for the Optical Conductivity,” Physical Review, Vol. 64, No. 15, 2001, Article ID: 155106. doi:10.1103/PhysRevB.64.155106

[35] J. von Neumann and O. Morgenstern, “Theory of Games and Economic Behavior,” 60th-Anniversary Edition, Princeton University Press, Princeton, 2004.

[36] W. M. Saslow, "An Economic Analogy to Thermodynamics," American Journal of Physics, Vol. 67, No. 12, 1999, p. 1239. doi:10.1119/1.19110

[37] A. Jakimowicz, "Catastrophes and Chaos in Business Cycle Theory,” Acta Physica Polonica A, Vol. 117, No. 4, 2002, p. 640. 